factors and sources of resiliency in LGBTQ populations. Culturally competent care is integral to psychiatric treatment of older LGBTQ adults.

\title{
Note:
}

This abstract was presented at the American Association of Geriatric Psychiatry 2021 Annual Meeting.

\section{4 - Validation of a new cognitive screening tool, the Brain Health Test-7, for identification of mild cognitive impairment and early dementia in 3 differentkinds of hospital settings}

Meng-Shiuan Shie ${ }^{1}$, Mei Xian Loi ${ }^{4}$, His-Chung Chen ${ }^{1,2}$, Ming-Hsien Hsieh ${ }^{1,2}$, Yi-Ting Lin ${ }^{1,2}$, Chen-Chung Liu ${ }^{2,3}$, PeiNing Wang ${ }^{5,6,7}$, Jiahn-Jyh Chen ${ }^{8}$, Cheng- Sheng Chen ${ }^{9,10}$, Chih-Cheng Hsu ${ }^{11}$, Tzung-Jeng Hwang ${ }^{1,2}$

${ }^{1}$ Department of Psychiatry, National Taiwan University Hospital, Taipei,Taiwan.

2 Department of Psychiatry, College of Medicine, National Taiwan University,Taipei, Taiwan.

${ }^{3}$ Department of Psychiatry, Hsin-Chu Branch, National Taiwan University,Taipei, Taiwan.

${ }^{4}$ Department of Psychiatry, Far Eastern Memorial Hospital, New Taipei City,Taiwan.

${ }^{5}$ Department of Neurology, Neurological Institute, Taipei Veterans GeneralHospital, Taipei, Taiwan

${ }^{6}$ Brain Research Center, National Yang Ming Chiao Tung University, Taipei,Taiwan.

${ }^{7}$ Aging and Health Research Center, National Yang Ming Chiao TungUniversity, Taipei, Taiwan.

${ }^{8}$ Department of Geriatric Psychiatry, Taoyuan Psychiatric Center, Minitry ofHealth and Welfare, Taoyuan, Taiwan

9 Department of Psychiatry, Chung-Ho Memorial Hospital, Kaohsiung MedicalUniversity, Kaohsiung, Taiwan

10 Department of Psychiatry, School of Medicine, Kaohsiung MedicalUniversity, Kaohsiung, Taiwan.

${ }^{11}$ Division of Geriatrics and Gerontology, Institute of Population HealthSciences, National Health Research Institutes, Taiwan.

\section{Background}

The Brain Health Test-7 (BHT-7) is a revised tool from the original BHT, containing more tests about frontal lobe function. It was developed with theaim of identifying patients with mild cognitive impairment $(\mathrm{MCl})$ and early dementia.

\section{Research objective}

Here we report the validity of the BHT-7 versus the Mini-Mental State Examination (MMSE) and Montreal Cognitive Assessment (MoCA) in differentpsychiatry or neurology clinics.

\section{Methods}

Patients with memory complaints were recruited in this study from the outpatient clinic of psychiatry or neurology in 3 different kinds of hospitals. Allpatients underwent the evaluation of the BHT-7, MMSE, MoCA, and clinical dementia rating (CDR). The clinical diagnosis (normal, $\mathrm{MCl}$, dementia) was made by consensus meeting, taking into account all available data.

Demographic data and the scores of the MMSE, MoCA, and BHT-7 between groups were compared. Logistic regression was adopted for analysis of optimal cutoff values, sensitivity, specificity, positive predictive value (PPV), negative predictive value (NPV), receiver operating characteristic (ROC) curve, and the area under the ROC curve (AUC).

\section{Results}

We enrolled a total of 1090 subjects (normal 402, MCl 317, dementia 371); of them, 705 (64.7\%) were female. There was a statistically significant differencein age, years of education, and 3 cognitive test scores among the 3 groups. 
Compared with the MMSE and MoCA, the BHT-7 performed slightly betterthan MMSE and MoCA in differentiating $\mathrm{MCl}$ or dementia from the normalcontrols (Table 1). For BHT7 , the cutoff point was 17 between normal andMCl, and 14 between normal and dementia. These cutoff points for BHT-7were consistent through 3 different clinical settings, but inconsistent for MMSE and MoCA. The testing time for the BHT-7 was about 5-7 minutes, shorter than that of the MMSE and MoCA.

\section{Conclusion}

Compared with MMSE and MoCA, the BHT-7 showed slightly better performance in differentiating normal from $\mathrm{MCl}$ or dementia subjects. The testing time for the BHT-7 was shorter, and its cutoff points were consistent through different outpatient clinic settings. The results support that BHT-7 is auseful cognitive screening tool for $\mathrm{MCl}$ or early dementia in various hospital settings.

Table 1: Comparisons of the performance of BHT-7, MMSE, MoCA

\begin{tabular}{cllllll}
\hline & AUC & cutoff & SEN & SPE & PPV & NPV \\
\hline Normal vs. MCI & & & & & & \\
BHT-7 & 0.8532 & $\leqq 17$ & 0.8170 & 0.7413 & 0.7135 & 0.8371 \\
MMSE & 0.8061 & $\leqq 27$ & 0.7950 & 0.6883 & 0.6684 & 0.8091 \\
MoCA & 0.8316 & $\leqq 25$ & 0.8202 & 0.6791 & 0.6684 & 0.8273 \\
\hline Normal vs. Dementia & & & & & & \\
BHT-7 & 0.9848 & $\leqq 14$ & 0.9434 & 0.9602 & 0.9563 & 0.9484 \\
MMSE & 0.9693 & $\leqq 24$ & 0.8895 & 0.9626 & 0.9565 & 0.9040 \\
MoCA & 0.9768 & $\leqq 21$ & 0.9245 & 0.9428 & 0.9372 & 0.9312 \\
\hline Normal vs. MCI+ Dementia & & & & & & \\
MHT & 0.9241 & $\leqq 16$ & 0.8372 & 0.8458 & 0.9028 & 0.7522 \\
MOCA & 0.8941 & $\leqq 25$ & 0.7282 & 0.9152 & 0.9365 & 0.6625 \\
\hline
\end{tabular}

545 - Exploratory factor analysis of the Rowland Universal Dementia Assessment Scale.

Authors:

Garcia-Casal, J. Antonio ${ }^{1}$

Coelho de Cunha Guimarães, Natacha

Díaz Mosquera, Sofía ${ }^{3}$

Alvarez Ariza, María ${ }^{4}$

Mateos Álvarez, Raimundo ${ }^{5}$

${ }^{1}$ Clinical Psychologist, Servicio Navarro de Salud, IdiSNA, Pamplona, Spain.

${ }^{2}$ Primary Care Physician, Granadilla de Abona Health Center, Tenerife, Spain.

${ }^{3}$ Helicopter and ambulance emergency physician, Galician Public Health Emergency Foundation, Santiago de Compostela, Spain.

${ }^{4}$ Department of Psychiatry, Vigo Health Area, Hospital Álvaro Cunqueiro, Spain Translational Neuroscience Group, Galicia Sur Health Research Institute, Spain. 